\title{
Effect of Ultraviolet Radiations on Medium and High Voltage Cables Insulation Properties
}

\author{
Abdallah Hedir $^{* 1}$, Mustapha Moudoud ${ }^{* 2}$ \\ * Departement of Electrical Engineering, Mouloud Mammeri University of Tizi-Ouzou \\ BP 17 RP, 15000 Tizi-Ouzou, Algeria \\ 1 abdallahhedir@yahoo.fr \\ 2 m_moudoud@yahoo.fr
}

\begin{abstract}
Under operating conditions effect, insulated power cables can undergo critical degradations. Ultraviolet (UV) radiations are one of the most destructive constraints which affect the properties of the material used for insulation. Because of its good properties, crosslinked polyethylene (XLPE) is widely used in medium voltage (MV) and high voltage (HV) cables insulation. Regardless of its excellent performances, XLPE can degrade when exposed to UV. The objective of this work is to report experimental results concerning the effect of accelerated UV ageing on the properties of XLPE insulation. For this purpose, dielectric characterization, visual observations and scanning electron microscopy (SEM) analysis are performed to assess the extent of ageing. Obtained results show that UV ageing affects greatly the XLPE insulation. So, an evolution in the dielectric properties (dielectric constant, dissipation factor, dielectric loss index and AC volume resistivity), color change and deterioration of surface morphology with ageing time are noticed.
\end{abstract}

Keywords - Ultraviolet (UV) Radiations, Insulation, Crosslinked Polyethylene, UV Ageing, Dielectric Chracterisation.

\section{INTRODUCTION}

Crosslinked polyethylene (XLPE) is one of the polymeric materials used widely in the field of cable insulation [1]-[2]. The use of this polymer has boomed in recent years, thanks to its low cost, ease of manufacturing and its good electrical and mechanical properties [3]. The manufacturing technology of medium and high voltage cable consists of using several materials around the conductive core. The XLPE layer is often an intermediate insulation, but in some cases, it may be an external one. Compared to all polymers, XLPE can know crucial degradations under the effect of operating conditions in which it works. These conditions act directly on its reliability and lifetime. According to the literature, the ultraviolet exposure is one of the most destructive factors [4]-[5] of any electrical insulation, such as XLPE insulation. Almost all the works dedicated to the photooxidation phenomenon of polyolefins have been directed towards the study of the degradation of low density polyethylene, high density polyethylene and polypropylene [1]-[6]-[7]. However, there exist few works dedicated to the study of the photodegradation phenomenon of XLPE; this is due to its recent use in the area of electrical insulation. The sun is the most important source of light, the spectrum of sunlight penetrating the Earth's surface ranges from 290 to 3000nm [8]. The portion ranging from 290 to $400 \mathrm{~nm}$ represents the UV irradiation (UV-B and UV-A), is demonstrated to be the portion that is harmful tomost polymers [9]. According to previous works, many important issues have been discussed regarding the ultraviolet (UV) aging of polymers. The influence of this constraint on the polymer's lifetime received considerable interest in recent years [10][11]. Then it has been demonstrated that the photodegradation of polymers leads systematically to an evolution and change in morphology (chains scission, cross-linking and crystallization) and the formation and decomposition of hydroperoxydes [12]-[14]. All these chemical evolutions lead to the change of dielectric and mechanical properties of these materials [15]-[17]. Another area of interest is the change in appearance when polymers are exposed to UV radiation. The mechanism of yellowing remains always a matter of opinion that well-defined phenomenon. But a variety of chromophors have been proposed to be responsible for this phenomenon [18]. The aim of this work is to study the possible effects of accelerated aging under UV radiations on dielectric properties, appearance and surface morphology of XLPE insulation. This paper is organized as follows: Section II presents the experimental setup. In Section III, the obtained experimental results are illustrated and discussed. Section IV concludes this paper. 


\section{A. Samples Preparation}

\section{EXPERIMENTAL SETUP}

The XLPE plates have been obtained from granules of natural crosslinkable polyethylene compound LE4201R. The material contains dicumyl peroxide with concentration of $2 \%$ to generate cross-links. The granules are placed in molds and heated to $130^{\circ} \mathrm{C}$ at different pressures and for different time durations. This temperature mainained until fusion of the granules is complete. After the full merger, pressure is increased to 300 bars and the temperature to $180^{\circ} \mathrm{C}$ (curing temperature). These conditions $\left(180^{\circ} \mathrm{C}, 300 \mathrm{bars}\right)$ are maintained for two minutes in order to get a complete material reticulation. Finally the granules are cooled at $45^{\circ} \mathrm{C}$, and square plates of $130 \mathrm{~mm} \times 130 \mathrm{~mm}$ with $2 \mathrm{~mm}$ thickness of XLPE are obtained.

The electrical characteristics of the used material are as follows:

TABLE I Electrical Properties of the Used Material

\begin{tabular}{|c|c|c|}
\hline Property & $\begin{array}{c}\text { Typical } \\
\text { value }\end{array}$ & Test method \\
\hline $\begin{array}{c}\text { Dielectric Constant } \\
(50 \mathrm{~Hz})\end{array}$ & 2.3 & IEC 60250 \\
\hline $\begin{array}{c}\text { DC Volume Resistivity } \\
\text { POhm.cm }\end{array}$ & IEC 60093 \\
\hline $\begin{array}{c}\text { Dielectric Strength } \\
(50 \mathrm{~Hz})\end{array}$ & $>22 \mathrm{kV} / \mathrm{mm}$ & IEC 60243 \\
\hline
\end{tabular}

\section{B. UV Exposure}

The UV ageing is carried out using a cubic chamber $(80 \times 60 \times 60 \mathrm{~cm})$, designed and built specially for this study. The chamber is fabricated with Plexiglas and isolated with polystyrene from the outside; the inner faces are covered with aluminum foil (reflection coefficient of about 0.9 ) to avoid any loss of radiations emitted by the UV sources. The samples irradiation is accomplished using eight low-pressure vapor 36-watt fluorescent lamps. The spectrum of the sources is mostly in the UV-A region, emitting radiations with wavelength ranging from 350 to $400 \mathrm{~nm}$. The lamps provide also a minimum percentage of radiations in the UV-B region with wavelength ranging from 280 to $315 \mathrm{~nm}$. The samples-to-lamp distance is $10 \mathrm{~cm}$. Only one side of the samples is subjected to radiations. The total duration of exposure is 240 hours. The ageing is done under a temperature of $55 \pm 5^{\circ} \mathrm{C}$ without controlling the humidity.

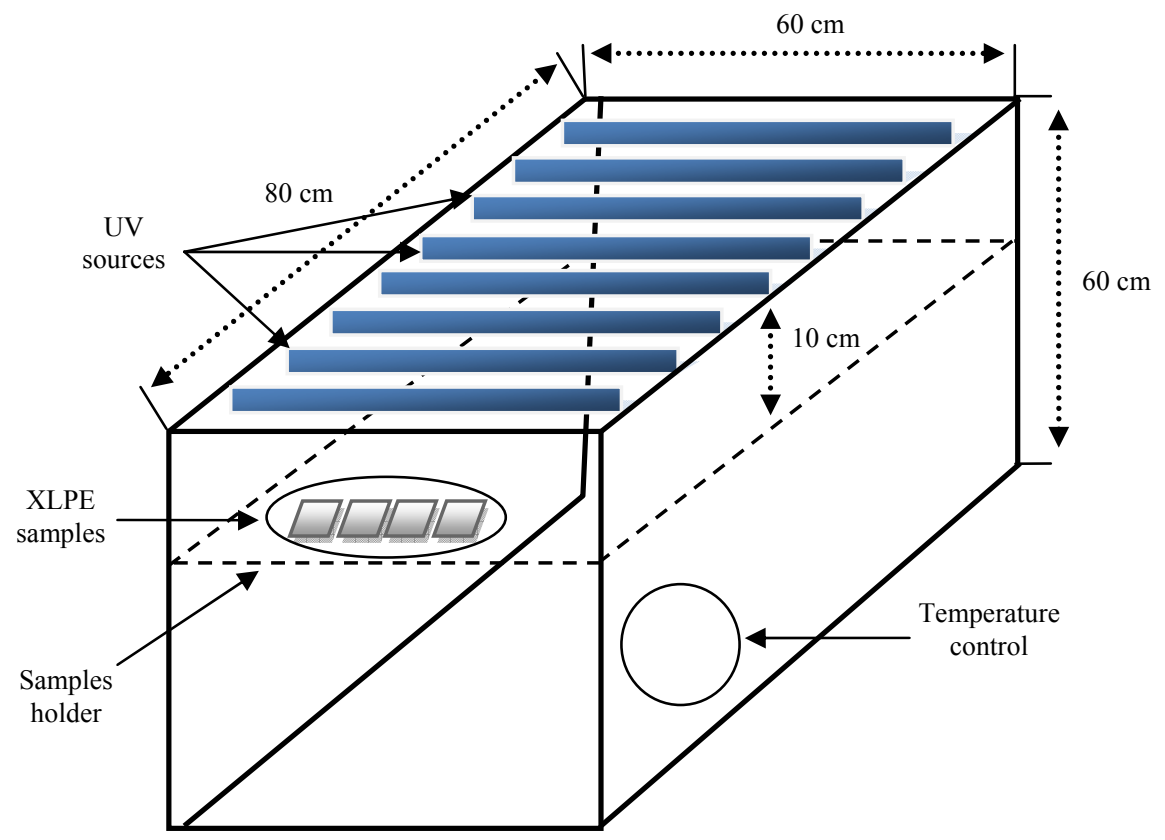

Fig.1.Schematic diagram of the UV radiation chamber.

\section{Dielectric Characterization}

The dielectric properties of XLPE' samples are measured and investigated using an LCR meter (Instek-LCR 817 type); able of measuring the properties of materials properties at frequencies ranging from 12 to $1000 \mathrm{~Hz}$, the measurement voltage of the apparatus did not exceed $2 \mathrm{~V}$. The LCR meter is connected with shortest possible 
cables to the measuring cell. This cell is composed of two bronze cylindrical electrodes. Relative Permittivity, dissipation factor and volume resistivity are measured at low voltage $(1 \mathrm{~V})$ with frequency ranging from 0.5 to $10 \mathrm{kHz}$.

Generally, the written permittivity in its complex form is given by:

$\varepsilon^{*}=\varepsilon^{\prime}-j \varepsilon^{\prime \prime}$

The real part $\varepsilon^{\prime}$ of the complex permittivity represents the relative permittivity or dielectric constant, it tells us about the storing charge ability of dielectrics in the presence of electric field. The relative permittivity depends on the specimen thickness and the geometry of the measuring electrodes and has the equation:

$\varepsilon^{\prime}=\frac{C . e}{\varepsilon_{0} \cdot S}$

where $C$ is the capacitance of the sample sandwiched between electrodes, $e$ is the spacing between electrodes which is equal to the sample thickness, $S$ is the electrode area, and $\varepsilon_{0}=8.85 \times 10^{-12} \mathrm{~F} . \mathrm{m}^{-1}$ is the vacuum permittivity. The imaginary part $\varepsilon$ " of the complex permittivity represents the dielectric loss index. The losses are caused mainly by the dipoles movement under the influence of an alternating electric field, and they are relative to the conductivity due to ions and to the dipole relaxation and polarization phenomena. The dissipation factor tan $\delta$ also called tangent of the angle $\delta$, which is the complementary angle of the phase difference between the voltage applied to the dielectric and the resulting current. In accordance with Debye theory, the dissipation factor is determined as:

$\tan \delta=\frac{\varepsilon^{\prime \prime}}{\varepsilon^{\prime}}$

The AC volume resistivity is obtained from the resistance given by the LCR meter, this resistivity has the equation:

$\rho=\frac{R \cdot S}{e}$

where $\mathrm{R}$ is the resistance given by the measuring apparatus, $S$ is the electrode area, and $e$ is the sample thickness.

\section{Visual Observation and SEM Analysis}

The visual observations are essentially based on the color change study of the samples subjected to UV radiations. In order to study the changes in morphology of XLPE aged under UV, micrographs of samples in different aging states were investigated on the surfaces by scanning electron microscopy (SEM). SEM has become a powerful technique in materials research [19]; it is based on the principle of electron interaction with matter. It allows obtaining images of the sample surface with high resolution and providing information about the morphology and the chemical composition of a solid object. In our work, the analysis is done with a $2000 \times$ magnification electron microscope (Phillips XL30-type) under a voltage of $20 \mathrm{kV}$.

\section{RESUlts AND ANALYSIS}

\section{A. Dielectric Proporeties}

\section{1) Dissipation Factor (Dielectric Factor)}

The evolution of the dissipation factor $\tan \delta$ versus frequency $f_{m}$ and ageing time $\tau$ is shown respectively in Fig.2 and Fig.3.

In Fig.2, it can be observed that tan $\delta$ presents descending curves for both before and after aging. Consequently, the $\tan \delta$ values decrease within increasing $f_{m}$, especially for low frequencies. It is also found that the dissipation factor has more pronounced values after aging. Therefore, it is noted that the tan $\delta$ of the unaged sample is small compared to those of aged samples. At $1 \mathrm{kHz}$ for example, tan $\delta$ of the unaged sample is 0.0261 , while it grows to 0.0321 afetr 100 hours and then to 0.0484 after 200 hours of exposure.

It can be seen in Fig. 3 that $\tan \delta$ exhibits a non-monotonic variations, with occasional peaks. So, after a period of relative stability at the beginning of aging, followed by a rapid increase where tan $\delta$ reaches high values after a relatively short time $(50 \mathrm{hr})$. It starts a phase where $\tan \delta$ do not shows significant variations. After this phase, $\tan \delta$ exhibits a subsequent recovery.

The dielectric factor mainly depends on the electrical conductivity and loss polarization such as dipole polarization [20]. The number of charge carriers in the bulk material and the applied voltage frequency are two parameters which can influence electrical conductivity. 
The increase of $\tan \delta$ after aging (Fig.2) can be attributed to the introduction of more dissociative ions and incorporation of additional polar groups which act as charge carriers. The phenomenon of introduction and incorporation of contaminants (ions and polar groups) leads to the increase of: lossy polarization, electrical conductivity and dissipation factor.

The results shown in Fig. 3 can be explained as follows: The noted decrease of the dissipation factor at the beginning of exposure to UV can be attributed to the improvement of the XLPE insulation quality, indicating a drop in molecular mobility [21]. As it is known, $\tan \delta$ is strongly influenced by the oxidation phenomenon in the case of polyethylene [22]-[23]. Therefore, the growth of tan $\delta$ can be interpreted as the formation due to oxidation of electrically assymetric carbonyl compounds. These compounds often play a very important role in disruption of dielectric properties (increasing losses) [24]-[25].

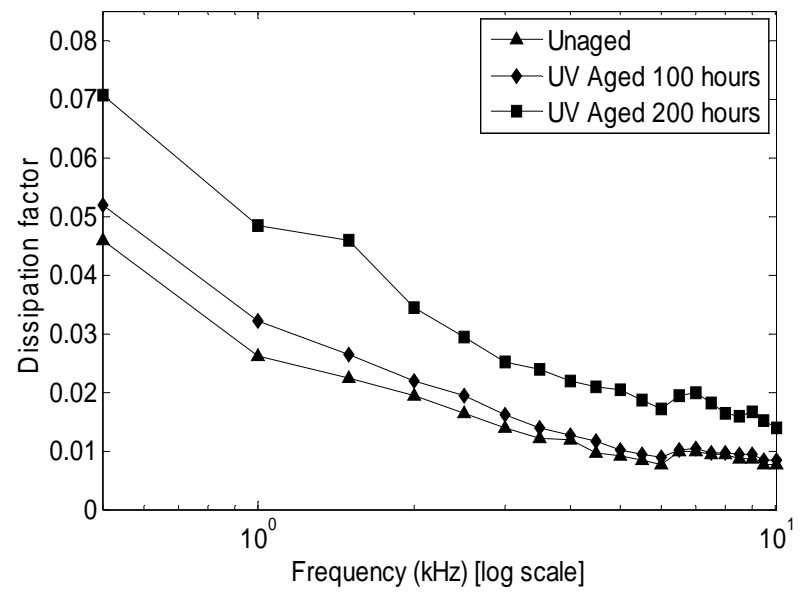

Fig.2. Dissipation factor of XLPE as a function of measurement frequency for different ageing times

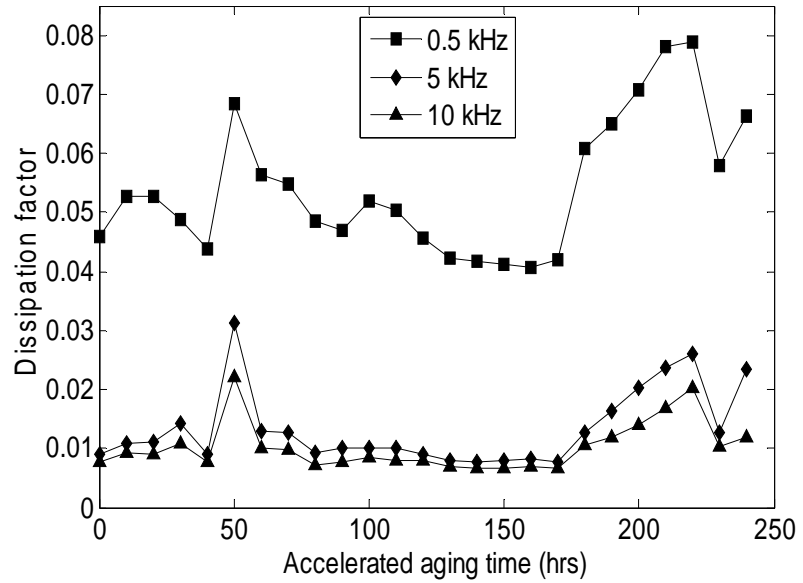

Fig.3. Dissipation factor of XLPE as a function of: UV exposure time for different frequencies

\section{2) Relative Permittivity}

The relative permittivity $\varepsilon$ as a function of frequency variation $f_{m}$, and ageing time $\tau$ are shown respectively in Fig.4 and Fig.5.

It can be seen from Fig. 5 that $\varepsilon$ decreases with increasing $f_{m}$, this decay is remarkably more important at low frequencies. In the other hand, $\varepsilon$ ' 'increases with increasing aging time $\tau$. At $1 \mathrm{kHz}$, for example, the value of $\varepsilon$ ' of the unaged sample is 2.5510 , while it grows to 2.6513 when the sample is subjected to UV for 100 hours and it increases to 2.7880 for 200 hours of aging.

Fig. 5 shows that $\varepsilon$ decreases at the beginning of aging, it presents its minimum after a relatively short time (20hr), the decrease is followed by an unceremonious increase where $\varepsilon$ reaches high values. After that, it begins a phase where after a decay, $\varepsilon$ remains relatively stable but interfered by some peaks (120 and 140 hrs). This phase is followed by an increase in relative permittivity value. This behavior, although repeatable, requires further work to understand.

As it is known, relative permittivity is determined by several phenomena arising in the bulk of the material, such as relaxation and polarization. The permittivity is governed by the number of orientable dipoles and their ability to orient themselves under an applied electric field [20]-[26]. Then, the results shown in Fig.4, where a 
dependence of relative permittivity on frequency is ascertained, can be assigned to the dielectric relaxation phenomenon. So when a low frequency electric field is applied, it results a high permittivity value. This value can be attributed to the orientation of all the free dipolar groups whether big or small at the pace of the applied field. As the electric field frequency increases, dipolar groups' orientation, especially the biggest becomes difficult, involving an attenuation of the dipoles' contribution to relative permittivity. This attenuation leads to a continuous decrease of the permittivity. The pronounced changes after aging are caused by the easy diffusion of oxygen, this which implies an increase in the speed of oxidation, and formation of polar groups such as hydroxyl and carboxyl. These polar groups will contribute to the total polarization and thus cause an increase of the dielectric constant (relative permittivity) value.

To illustrate the shape of the curves described in Fig.5, the results and structural changes induced by the photooxidation phenomenon can be correlated. The decrease of $\varepsilon^{\prime}$ at the beginning of aging can be explained by the rearrangement of the molecular structure and the crosslinking phenomenon. The increase in the dielectric constant value after a relatively short period is due to the shrinkage experienced by the material upon exposure to UV radiation, thus to the increase of its volume. From a microscopic point of view, an alternative explanation can be given for this increase, considering the phenomenon of shocks sustained by dipoles and the associated relaxation time. Thus, the increase is due to the change of this time representing the average period between two successive shocks.

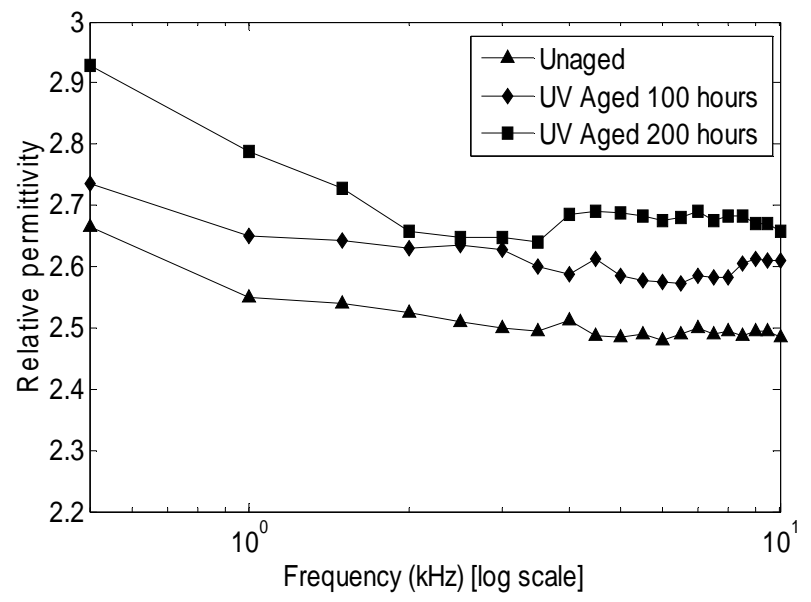

Fig.4. Relative permittivity of XLPE as a function of measurement frequency for different ageing times

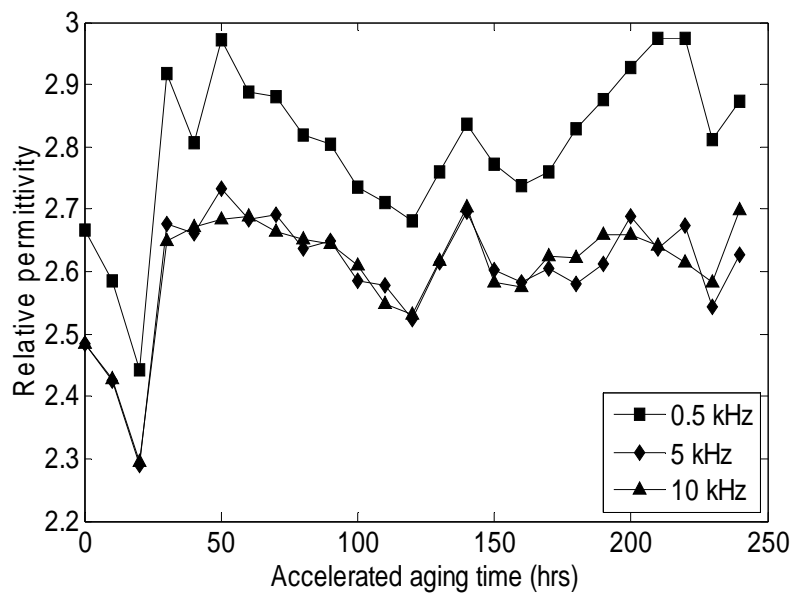

Fig.5. Relative permittivity of XLPE as a function of UV exposure time for different frequencies

3) Dilectric Loss Index

The results given in Fig.6 and Fig.7 display respectively the variation of the dielectric loss index $\varepsilon$ " versus frequency $f_{m}$ and aging time $\tau$.

It can be seen in Fig.6 that $\varepsilon$ " presents decreasing curves in virgin and aged states, with remarkably higher values after aging. Again at $1 \mathrm{kHz}$, the dielectric loss index of the unaged sample is 0.0665 , while it increases to 0.0851 when the sample is aged under UV for 100 hours and it grows and reaches a value of 0.1349 for 200 hours of aging.

As it is shown in Fig.7, the dielectric loss index $\varepsilon$ "follows the dissipation factor (tan $\delta$ ) variations (Fig.2). Then, it can be seen that $\varepsilon$ " remains practically constant at the beginning of aging. After that, a brief increase after $50 \mathrm{~h}$ 
of aging where $\varepsilon "$ reaches an elevated value arises. After this increase, the $\varepsilon$ " curve is experiencing a sudden decay, followed by a phase where $\varepsilon$ " remains constant. After this phase, $\varepsilon$ " knows a second growth and reaches very high values.

As it is known, dielectric loss index is the product of the relative permittivity and dissipation factor. The use of this property has the advantage of bringing together in one term two characteristics of the material. Thus, the evolution of the dielectric loss index tells us about the power lost in the polymer. Therefore, any increase or decrease of the dielectric loss index corresponds to an increase or decrease of the power lost in the material. The increase of dielectric loss index with ageing ageing shown in Fig. 8 can be explained by the deterioration in the material's quality. This derterioration of properties must be caused by Joule heating in the bulk induced by the increase of dielectric loss factor. The presence of relaxation peak showed in Fig.6 reveals the existence of several absorption bands [27].

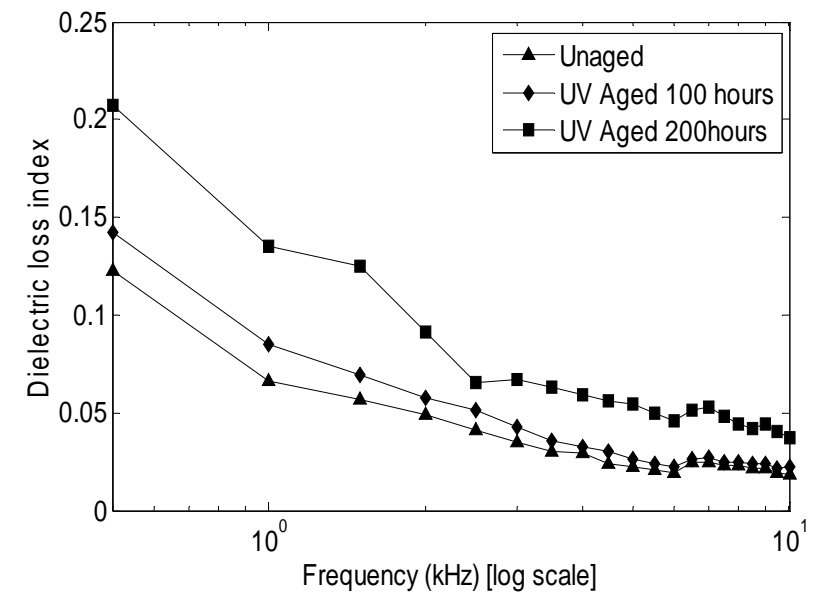

Fig.6. Dielectric loss index of XLPE as a function of measurement frequency for different ageing times

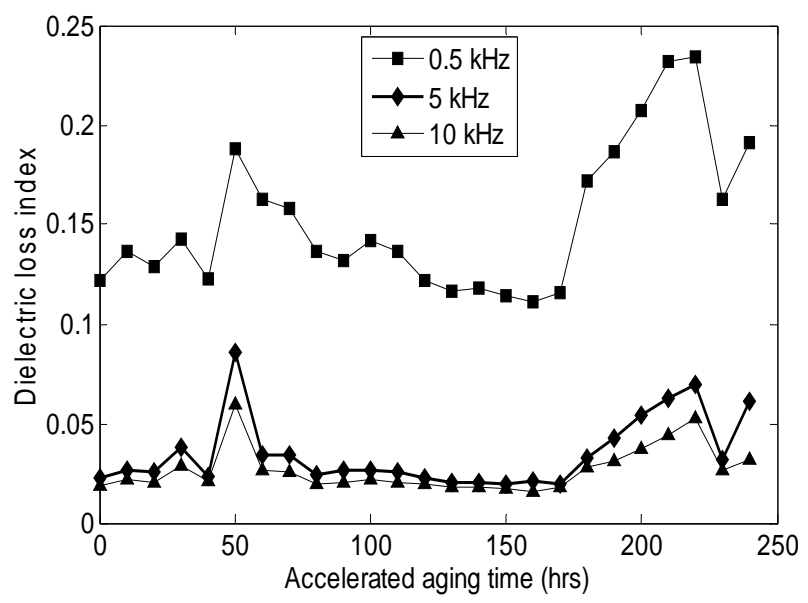

Fig.7. Dielectric loss index of XLPE as a function of UV exposure time for different frequencies

4) Volume Resistivity

The evolution of AC volume resistivity versus frequency variation $f_{m}$ and ageing time $\tau$ is shown in Fig.8 and Fig.9, respectively.

To a certain extent, the $\mathrm{AC}$ volume resistivity constitutes a diagnostic tool for aging characterization. It can represent the insulation performance of the material.

As in the case of the others dielectric parameters studied previously, it can be observed in Fig.8 that the AC volume resistivity decreases when $f_{m}$ increases. This decrease appears remarkably at low frequencies.

Qualitatively, it can be seen in Fig.9 that the AC volume resistivity of XLPE shows slight and non monotonic variations with respect to UV exposure time. These variations are more pronounced at low frequencies. Specifically for $0.5 \mathrm{kHz}$, AC volume resistivity increases from about $4.07 \times 10^{8} \Omega \mathrm{cm}$ to $6.3 \times 10^{8} \Omega \mathrm{cm}$ value at the beginning of UV exposure and reaches the value of $4.19 \times 10^{8} \Omega \mathrm{cm}$ after 240 hours of aging. Generally, the $\mathrm{AC}$ volume resistivity remains relatively constant with UV exposure time. 


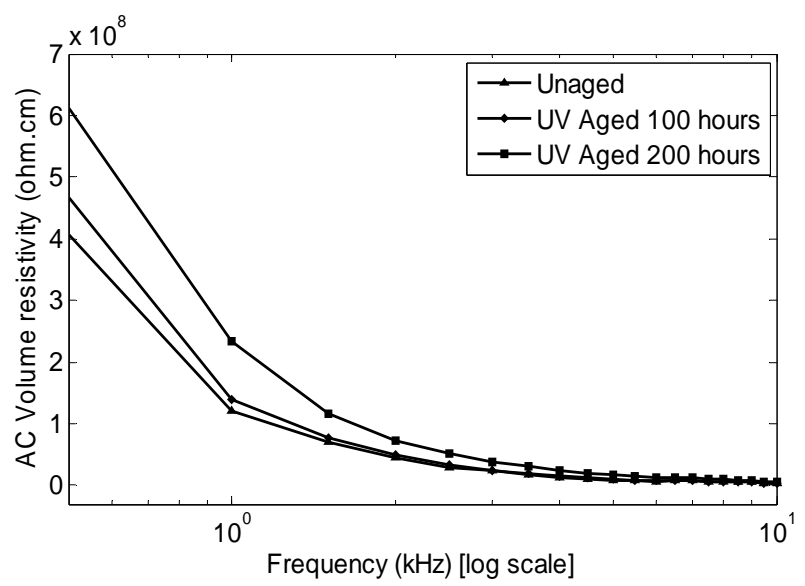

Fig.8. AC volume resistivity of XLPE as a function of measurement frequency for different ageing times

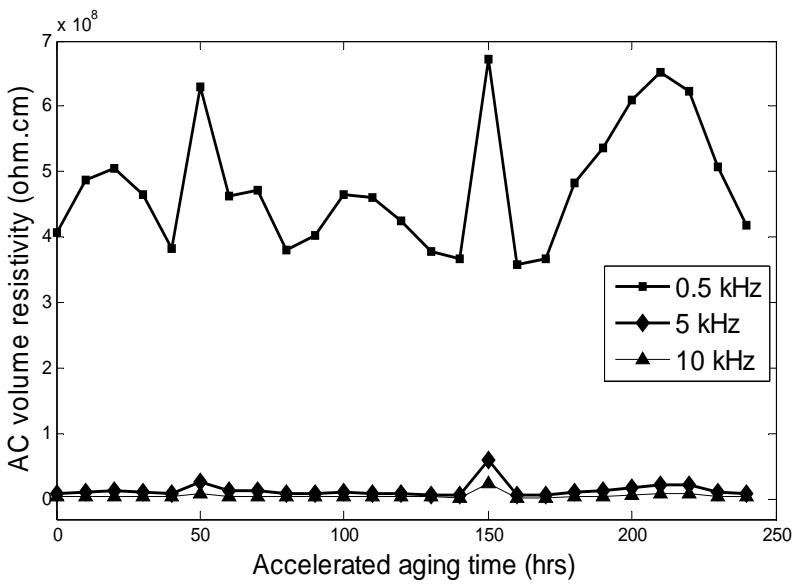

Fig.9. AC volume resistivity of XLPE as a function of UV exposure time for different frequencies

\section{B. Discoloration Due to UV Ageing}

Fig.10 shows the color change of here samples that were aged under UV radiations. As it can be noticed, the yellowing in exposed faces is linearly related to exposure time; the color varies from grey (unaged) to yellow (after 100h) and then turned to brown (after 200h). This change can be attributed to the photooxidation phenomenon that causes the formation of an oxidized layer on the surface of the substrate [28]. It was shown [29] that the photooxidation mechanism is purely a radical process, it includes three steps: initiation, propagation, and termination. All the reactions governing this phenomenon are shown below:

$$
\begin{aligned}
& \text { Polymer } \rightarrow R, H, R O O \quad \text { Initiation step } \\
& \mathrm{R}+\mathrm{O} \rightarrow \mathrm{ROO} \\
& \mathrm{ROO}+\mathrm{RH} \rightarrow \mathrm{ROOH} \\
& \mathrm{ROOH} \rightarrow \mathrm{RO}+\mathrm{OH} \\
& \text { Propagation step } \\
& 2 \mathrm{ROOH} \rightarrow \mathrm{R}+\mathrm{ROO}+\mathrm{OH} \\
& R^{\bullet}+R^{\bullet} \rightarrow R-R \\
& \mathrm{ROO} O^{\bullet}+\mathrm{ROO}^{\bullet} \rightarrow \mathrm{ROOR}+\mathrm{O}_{2} \\
& R O O^{\bullet}+R^{\bullet} \rightarrow R O O R \\
& R^{\bullet}+H^{\bullet} \rightarrow R H \\
& \text { Termination step }
\end{aligned}
$$

The ability of polymers to resist the penetration of UV radiation can be influenced by the presence of impurities or chromophors. The origin of chromophors can be catalyst residues, carbonyl groups, double bonds formed during material's shaping. In the initiation step, the radicals which initiate the process are formed in the presence of UV radiations and oxygen from chromophoric groups. This formation will take place by removing hydrogen from the polymeric chain. In propagation step, oxygen reacts with the free radicals to form hydroperoxydes radicals; these latter react with hydrogen and give hydroperoxydes. Hydroperoxydes being thermally and photochemically unstable will lead to polymer peroxy $(R O O)$, polymer alkyloxy $(R O)$ and hydroxyl $(O H)$ radicals. 
Finally, when two radicals combine in the propagation step, unstable or inactive products will be formed. These products may be aldehydes, ketones or acids.

The phenomenon of polymer's appearance change due to different parameters (electrical or environmental), was discussed by several researchers. The work of Moudoud and others [30] showed that the electrical ageing induces a change in the polymethyl methacrylate color. In the work concerning the styrene acrylonitrile surface degradation exposed to corona discharge, Handala and Lamrous [31] observed a change in the material appearance. They indicated that these evolutions are due to oxidation or functional group elimination along the polymer chain.

In this study, all the observations are in clear correlation with those encountered in the literature. The authors in [32] demonstrated that UV exposure darkens the color of some composites. In the study of the UV aging of certain polymers, the authors in [33] have shown that the surface of the material under investigation changes color in the first step of aging then remained almost unchanged. In [28], it is demonstrated that UV-exposure affects the appearance of polymers by changing the color of the side subjected to radiations. Elleder and Borovansky [34] indicated that the irradiation with UV light can induce strong yellow auto fluorescence due to products of oxidative degradation.
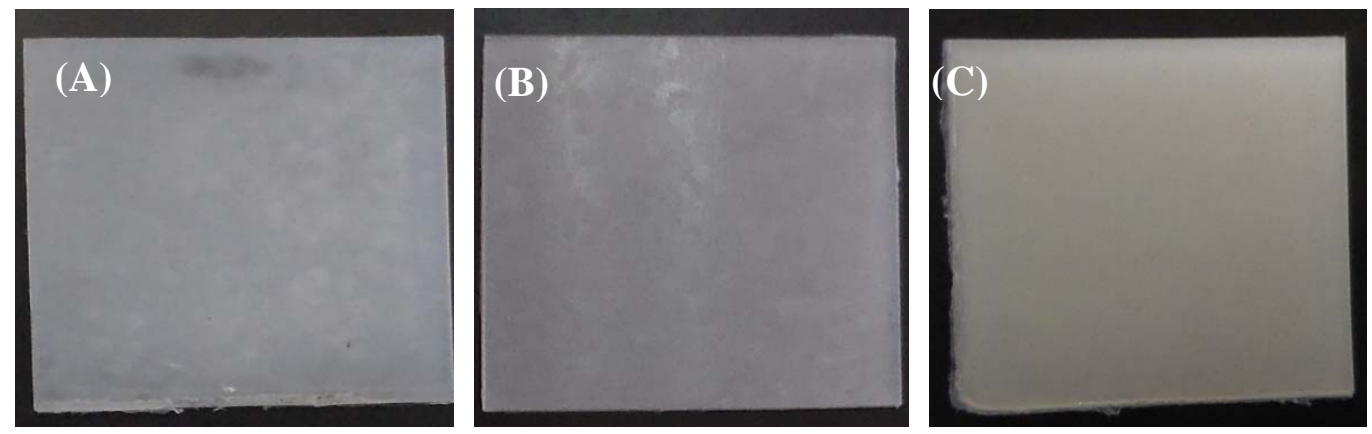

Fig.10. Color changes with duration of UV aging (A-Unaged, B-100 hours, and C-200 hours)

\section{Surface Morphology}

As mentioned in paragraph III-B, the polymers contain chromophoric groups, such as hydroxyl groups $(\mathrm{O}=\mathrm{H})$ and carbonyl groups $(\mathrm{C}=\mathrm{O})$. These chromophoric groups amust involve the absorption of photons or $\mathrm{UV}$ rays and are the reagents items that generate the photodegradation phenomenon [35]. Furthermore, Blaga [36] showed that the absorbed UV light energy causes the dissociation of bonds in the molecules of polymers constituents to produce free radicals as the primary photochemical products, when he studied the Durability of building materials and compounds. Subsequently, this event can lead to one or more of the following chemical changes: chain-scission, cross-linking, separation of small molecules, formation of double bonds in the main chain, depolymerization, and photohydrolysis (photolysis), etc. [36].

Fig. 11 shows the surface micrographs of XLPE samples obtained from scanning electronic microscope (SEM), of unaged and aged for 100 and 200 hours. The surface of the unaged sample showed in Fig.11.A, is smooth, homogeneous free from degradation and uniform. Fig.11.A and 11.C shows that significant damage occurs after ageing, these changes appear appropriately after 200hours of UV exposure, with creation of shallow microcracks, micro-cavities and embrittlement at the surface, resulting from degradation and blooming of volatile products. Similar microcracking phenomenon has been reported previously [3]-[37] upon UV-exposure. In these works, molecular chain scissions were found to occur on the polymer surface and to generate polymer radicals. These evolutions in samples may indicate that the deterioration of insulation is severe and may extend from surface, considering the translucent nature of the material [38]. They may be often accompanied by extensive deterioration in the mechanical properties of the materials, such as tensile strength, impact strength and elongation, all of which are important parameters in the performance of an insulating material.
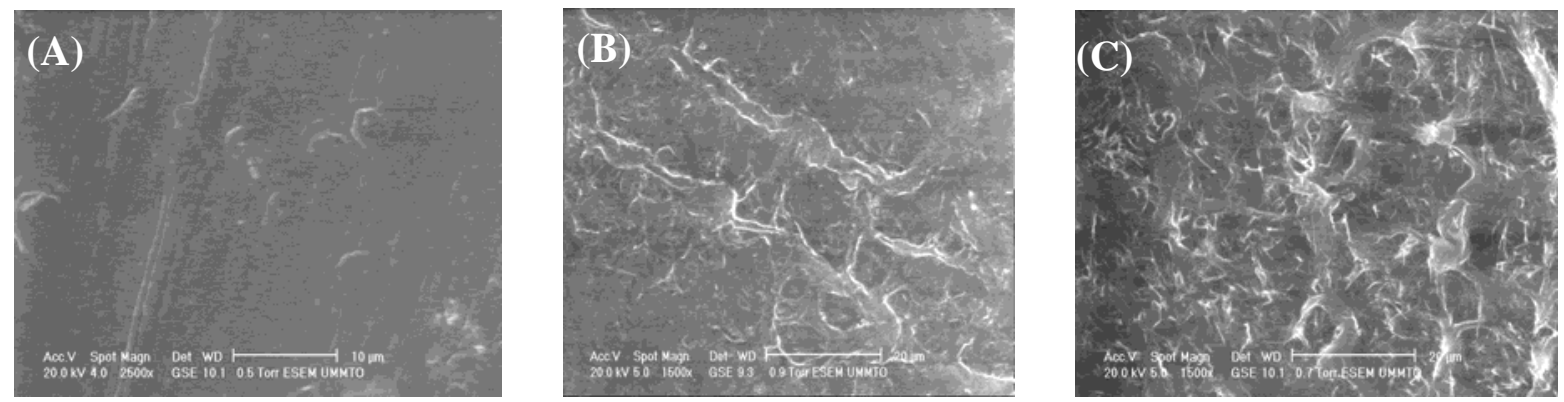

Fig.11. SEM micrographs (A-Unaged, B-100 hours, C-200 hours) 


\section{CONCLUSION}

The effect of UV-radiations on the XLPE insulation properties has been studied in this work. In this context dielectric characterization, visual observations and SEM analysis were performed. The conclusions of this paper can be summarized as follows:

- Dependence of dielectric properties on frequency was showed by dielectric characterization. Thus, it has been founded that all the properties decrease with frequency increasing.

- UV aging duration affect greatly the XLPE insulation. This degradation is characterized by an increase of all the dielectric properties (dielectric constant, dissipation factor, dielectric loss index), except of AC volume resistivity, which experienced a decrease. All these evolutions indicate deterioration in the insulating material quality.

- Visual appearance changes of the studied insulating material were observed. So, a color' change was noticed on the faces that were exposed submitted to UV radiations; the color varies from grey (unaged) to yellow (after 100 hours) and then tended to brown (200 hours).

- SEM micrographs showed damages caused by the exposure to UV radiations. Thus, microcracks, microcavities and embrittlement in the surface were created.

For more investigation in XLPE aging process under UV exposure, other analysis such as: Fourier transform infrared spectroscopy, differential scanning calorimetry and thermo-gravimetrical analysis will be performed.

\section{ACKNOWLEDGMENT}

The authors thank Enicab Biskra for helping to get XLPE plates.

\section{REFERENCES}

[1] M. Celina, G.A. George, "Characterisation and Degradation Studies of Peroxide and Silane Crosslinked Polyethylene", Polym Degrad Stab, vol. 48, pp. 297-312, January 1995.

[2] T.D. Martins, J.V. Gulmine, L. Akcelrud, R.G. Weiss, T.D.Z. Atvars, "Dependence of Relaxation Processes in a Low-density Polyethylene with Different Crosslink Densities Investigated by Fluorescence Spectroscopy", Polymer, vol. 47, pp. 7414-7424, October 2006.

[3] J.V. Gulmine, L. Akcelrud, "Correlations between Structure and Accelerated Artificial Ageing of XLPE", Eur Polym J, vol. 42, pp. 553-562, October 2006.

[4] I.M. Ward, D.W. Hadley, An Introduction to the Mechanical Properties of Solid Polymers, Wiley, Inc, 1993.

[5] J.F. Rabeck, Polymer Photodegradation-Mechanisms and Experimental Methods, Chapman\& Hall.1993.

[6] F. Gugumus, "Re-evaluation of the Stabilization Mechanisms of Various Stabilizers Classes", Polym Degrad Stab, vol. 39, pp. 117135, 1993, pp. 117-135.

[7] N.S. Allen, A. Chirinis-Padron, T.J. Henman, “The photo- stabilisation of polypropylene: A review”, Polym Degrad Stab, vol. 13, pp. 31-76, 1985.

[8] T. Xu, G. Li, S. Pang, "Effects of ultraviolet radiation on morphology and thermo-mechanical properties of shape memory polymer based syntactic foam", Composites Part A: Appl Sci Man, vol. 42, pp. 1525-1533, October 2011.

[9] J. Tochacek, Z. Vrátníčková, "Polymer life-time prediction: The role of temperature in UV accelerated ageing of polypropylene and its copolymers",Polym test, vol. 36, pp. 82- 87, June 2014.

[10] F. Delor, N. Barrois-Oudin, X. Duteurtre, C. Cardinet, J. Lemaire, J. Lacoste, "Oxidation of Rubbers Analysed by HATR/IR Spectroscopy", Polym Degrad Stab, vol. 62, pp. 395-401, November 1998.

[11] F.R. Flandrin, J.M. Widmaier, J.J. Flat, "Thermal ageing of polyurethane with hydrogenated polyisoprene soft segments", Polym Degrad Stab, vol. 57, pp. 59-67, July 1997.

[12] F. Carrasco, P. Pages, S. Pascual, X. Colom, “Artificial Aging of High-density Polyethylene by Ultraviolet Irradiation”, Eur Polym $J$, vol. 37, pp. 1457-1464, July 2001.

[13] A. Valadez-Gonzalez, J.M. Cervantes-Uc, L. Valeva, "Mineral Filler Influence on the Photo-oxidation of High Density Polyethylene: I Accelerated UV Chamber Exposure Test", Polym Degrad Stab, vol. 63, pp. 253- 260, February 1999.

[14] L. Doumingue, S. Mallarino, S. Cohendoz, X. Feaugas, J. Bernard, "Extrinsic Fluorescence as a Sensitive Method for Studying Photodegradation of High Density Polyethylene Part I", Curr Appl Phys, vol. 10, pp. 1211-1215, July 2010.

[15] T. Kurihara, T. Takahashi, H. Homma, T. Okamoto, "Oxidation of Cross-linked Polyethylene Due to Radiation-thermal Deterioration”, IEEE Trans Dielectr Electric Insul, vol. 1, pp. 878 - 887, June 2011.

[16] K. Anandakumaran, "Aging and Condition Monitoring Studies of Composite Insulation Cables Used in Nuclear Power Plants", IEEE Trans Dielectr Electric Insul, vol. 14, pp. 227- 237, February 2007.

[17] T.B. Zhao, R.A. Bernstorf, "Aging Tests of Polymeric Housing Materials for Non-Ceramic Insulator", IEEE Electric Insul Mag, vol. 14, pp. $26-33$, March/April 1998.

[18] J. Kubica, B. Waligora, "The Effect of Carbonyl Compounds on Photo-oxidation in Polystyrene films", Eur Polym J, vol.13, pp. 325$329,1977$.

[19] A.M. Nóbrega, M.L. Barreira-Martinez, A.A. Alencar de Queiroz, "Investigation and Analysis of Electrical Aging of XLPE Insulation for Medium Voltage Covered Conductors Manufactured in Brazil", IEEE Trans Dielectr Electric Insulation, vol. 20, pp. 628- 640, April 2013

[20] B.X. Du, J. Li, "Electrical and Mechanical Ageing Behaviors of Used Heat-Shrinkable Insulation Tubes", IEEE Trans Dielectr Electric Insulation, vol. 21, pp. 1875- 1881, August 2014.

[21] A. Kumar, S. Commereue, V. Verney, "Ageing of Elastomers: A Molecular Approach Based on Rheological Characterisation", Polym Degrad Stab, vol.85, pp. 751-757, August 2004.

[22] N. Shimizu, N. Hirano, K. Horii, "diagnosis of oxidation in xlpe by $\tan \delta$ characteristics", the $3^{\text {rd }}$ IEEE International Conference on Conduction and Breakdown in Solid Dielectrics, 3-6 july, 1989, Trondheim, Norway.

[23] N. Hirano, T. Tsujimura, N. Shimizu, K. Horii, "Diagnosis of the aged xlpe cable using frequency and temperature characteristics of $\tan \delta$. II", the $21^{\text {st }}$ IEEE, Symposium on Electrical and Insulating Materials, September 26, 1988, Japan.

[24] J. Verdu, Oxidative Ageing of Polymers, ISTE Ltd and John Wiley \& Sons Inc., 2012. 
[25] A. Motori, G.C. Montanari, S. Gubanski, "Low-Frequency Dielectric Properties for Diagnosis of Aging in Polymeric Cables", J Appl Polym Sci, vol. 59, pp. 1715-1724, March 1996.

[26] A. Livi, V. Levita, P. A. Rolla, "Dielectric Behavior at Microwave Frequencies of an Epoxy Resin during Crosslinking", J Appl Polym Sci, vol. 50, pp. 1583-1590, December 1993.

[27] J.C. Dubois, Plastiques. « Propriétés Diélectriques, Techniques de l’Ingénieur », A3140, August 1984, pp. 1-19.

[28] A. Boubakri, N. Guermazi, K. Elleuch, H.F. Ayedi, "Study of UV-aging of Thermoplastic Polyurethane Material", Mater: Sci \& Eng: A, vol. 527, pp. 1649-1654, March 2010.

[29] J.F. Rabek, B. Ranby, "Studies on the Photooxidation Mechanism of Polymers .I. Photolysis and Photo-oxidation of Polystyrene", $J$ Polym Sci: Polymer Chemistry Edition, vol. 12, pp. 273-294, February 1974.

[30] M. Moudoud, O. Lamrous, S. Diaham, M. Megherbi, "Electrical ageing effects on dielectric properties of polymethyl methacrylate", Eur Phys J Appl Phys, vol. 64, pp. 30201_p1-30201_p6, December 2013.

[31] M.A. Handala, O. Lamrous, "Surface degradation of styrene acrylonitrile exposed to corona discharge", Eur Tran on Electr Power, vol. 18, pp.495-505, July 2008.

[32] R.S.C. Woo, Y. Chen, H. Zhu, J. Li, J.K. Kim, C.K.Y. Leung, "Environmental Degradation of Epoxy-Organoclay Nanocomposites due to UV Exposure. Part I: Photodegradation", Compos Sci Technol, vol.67, pp. 3448-3456, December 2007.

[33] Q. Zhao, X. Li, J. Gao, "Aging of ethylene-propylene-diene monomer (EPDM) in artificial weathering environment", Polym Degrad Stab, vol. 92, pp. 1841-1846, October 2007.

[34] M. Elleder, J. Borovansky. "Auto fluorescence of melanins induced by ultraviolet radiation and near ultraviolet light - A histochemical and biochemical study". Histochemical J, vol. 33, pp 273-281, May 2001.

[35] B. Ranby, "Basic reactions in the photodegradation of some important polymers". J Macr Sci, Part A: Pure and Applied Chemistry, vol. 30, pp. 583-594, 1993.

[36] A. Blaga, "Durability of building materials and compounds". ASTM, Philadelphia: ASTM STP 691, 1980. pp. 827-837.

[37] C.N. Cascaval, C. Ciobanu, D. Rosu, L. Rosu, "Polyurethane epoxy maleate of bisphenol A semi-interpenetrating polymer networks", J Appl Polym Sci, vol. 83, pp. 138-144, January 2002.

[38] G. N. Wu, J. D. Wu, L. R. Zhou, B. Gao, K. Zhou, X. X. Guo and K. J. Cao, "Microscopic View of Aging Mechanism of Polyimide Film under Pulse Voltage in Presence of Partial Discharge", IEEE Trans Dielectr Electric Insul, vol. 17, pp. 125- 132, February 2010.
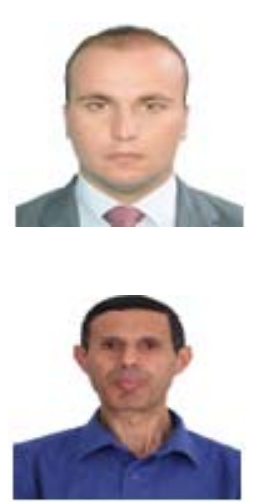

\section{AUTHOR PROFIL}

Abdallah Hedir was born in Tizi-Ouzou, Algeria, on June 29, 1988. He received his MSc in Electrical Engineering from the Mouloud Mammeri University of Tizi Ouzou, Tizi-Ouzou, Algeria in 2012. He is currently a Ph.D. student at the Mouloud Mammeri University of Tizi-Ouzou. His research activities concern the study of the electrical constraints and environmental parameters effects on the electrical insulation properties.

Mustapha Moudoud was born in 1964. He received the Engineer, magister and Ph.D. Degrees from Tizi-Ouzou University in 1988, 1997 and 2010, respectively. Currently, he is a lecturer in Tizi-Ouzou University. His research interests are in ageing effects on the dielectric characteristics of insulating polymers. 